\title{
Passive language mapping combining real-time oscillation analysis with cortico-cortical evoked potentials for awake craniotomy
}

\author{
Yukie Tamura, MD, ${ }^{1}$ Hiroshi Ogawa, MD, ${ }^{1}$ Christoph Kapeller, MSc, ${ }^{2}$ Robert Prueckl, MSc, ${ }^{2}$ \\ Fumiya Takeuchi, PhD, ${ }^{3}$ Ryogo Anei, MD, PhD, ${ }^{1}$ Anthony Ritaccio, MD, PhD, ${ }^{4}$ \\ Christoph Guger, PhD, ${ }^{2}$ and Kyousuke Kamada, MD, PhD'1
}

\begin{abstract}
${ }^{1}$ Department of Neurosurgery and ${ }^{3}$ Center for Advanced Research and Education, School of Medicine, Asahikawa Medical University, Hokkaido, Japan; ${ }^{2}$ Guger Technologies OG, Graz, Austria; and ${ }^{4}$ Department of Neurology, Albany Medical Center, Albany, New York
\end{abstract}

\begin{abstract}
OBJECTIVE Electrocortical stimulation (ECS) is the gold standard for functional brain mapping; however, precise functional mapping is still difficult in patients with language deficits. High gamma activity (HGA) between 80 and $140 \mathrm{~Hz}$ on electrocorticography is assumed to reflect localized cortical processing, whereas the cortico-cortical evoked potential (CCEP) can reflect bidirectional responses evoked by monophasic pulse stimuli to the language cortices when there is no patient cooperation. The authors propose the use of "passive" mapping by combining HGA mapping and CCEP recording without active tasks during conscious resections of brain tumors.
\end{abstract}

METHODS Five patients, each with an intraaxial tumor in their dominant hemisphere, underwent conscious resection of their lesion with passive mapping. The authors performed functional localization for the receptive language area, using real-time HGA mapping, by listening passively to linguistic sounds. Furthermore, single electrical pulses were delivered to the identified receptive temporal language area to detect CCEPs in the frontal lobe. All mapping results were validated by ECS, and the sensitivity and specificity were evaluated.

RESULTS Linguistic HGA mapping quickly identified the language area in the temporal lobe. Electrical stimulation by linguistic HGA mapping to the identified temporal receptive language area evoked CCEPs on the frontal lobe. The combination of linguistic HGA and frontal CCEPs needed no patient cooperation or effort. In this small case series, the sensitivity and specificity were $93.8 \%$ and $89 \%$, respectively.

CONCLUSIONS The described technique allows for simple and quick functional brain mapping with higher sensitivity and specificity than ECS mapping. The authors believe that this could improve the reliability of functional brain mapping and facilitate rational and objective operations. Passive mapping also sheds light on the underlying physiological mechanisms of language in the human brain.

http://thejns.org/doi/abs/10.3171/2015.4.JNS15193

KEY WORDS awake craniotomy; corticocortical evoked potentials; electrocorticography; high gamma activity; language; electrocortical stimulation; diagnostic and operative techniques

$\mathrm{W}$ HEN brain lesions are located in expressive areas, particularly those close to language-related structures, it is necessary to perform careful functional mapping to preserve expressive function while ensuring maximal lesion resection. However, although several epicenters and subcortical fibers are known to be involved, the complexity of the human language system means that completely accurate monitoring and mapping of language function cannot be assured.

Over recent decades, noninvasive functional MRI (fMRI) has been developed, which allows blood oxygen level-dependent visualization of activated brain regions. ${ }^{2,10,11,25}$ However, it has been shown that fMRI leads to the inclusion of many subsidiary cortical areas that

ABBREVIATIONS BIS = bispectral index; CCEP = cortico-cortical evoked potential; ECOG = electrocorticography; ECS = electrocortical stimulation; ERD, ERS = eventrelated desynchronization, event-related synchronization; fMRI = functional MRI; HGA = high gamma activity; PN = picture naming; WR = word reading. ACCOMPANYING EDITORIAL See pp 1577-1579. DOI: 10.3171/2015.6.JNS15927.

SUBMITTED January 28, 2015. ACCEPTED April 23, 2015.

INCLUDE WHEN CITING Published online March 18, 2016; DOI: 10.3171/2015.4.JNS15193. 
are not required for specific brain function. In particular, higher-order cognitive functions, such as language, appear to have wider supplementary areas when comparing fMRI results with electrocortical stimulation (ECS) mapping. ${ }^{18,28,34}$ Research into functional brain imaging has also focused on illustrating the subcortical fibers required for language function, and tractography has now become a standard procedure for visualizing the major axonal fascicles of interest by calculating the diffusion tensor values of each pixel.,14 Previous reports have described integrated tractography of arcuate fascicles into a neuronavigation system and confirmed the integrated fibers by ECS..$^{15,16}$ Despite this, it remains impractical to consistently extract the arcuate fascicles by tractography because there are no anatomical landmarks for the tracking initiation points and the fiber is closely packed with the corticospinal tract in the frontotemporal region. Given these anatomical and technical limitations, ECS during awake craniotomy remains the gold standard in language mapping..$^{70-32}$ However, technical difficulties still limit its use, including the need for patient cooperation, identifying optimal stimulation sites, ECS-related side effects, and the inherent seizure risk. Therefore, we have focused on extracting language-related activity in real time by recording electrophysiological signals directly from the brain surface instead of by ECS.

The dynamics of oscillatory neuronal activity on electrocorticography $(\mathrm{ECoG})$ have been proposed as potential neurophysiological indicators. Among these oscillatory changes, augmentation of high gamma activity (HGA) from approximately 60 to $140 \mathrm{~Hz}$ was assumed to reflect localized cortical processing. Crone et al. constructed HGA maps of language-related functions in patients performing word-reading tasks, which were confirmed by ECS. ${ }^{6}$ Therefore, HGA sheds light on the optimal signal for accurate functional mapping. We have reported a clinical user interface for real-time HGA mapping in awake craniotomy, with combinations of various language tasks inducing HGA distribution in expressive language and motor cortices. ${ }^{24}$ However, this revealed basic issues for patients with motor-dominant or conduction aphasia who could not perform language-execution tasks. Indeed, such patients are candidates for neither real-time HGA analysis nor routine ECS mapping, leaving a group with an unmet need.

Recently, cortico-cortical evoked potentials (CCEPs) have received strong attention for monitoring subcortical fiber functions. ${ }^{38}$ With stimulation to expressive sites, CCEPs appear in functionally connected brain cortices without any specific tasks. Because the evoked potentials are bidirectional responses, we expected that it would be possible to record CCEPs by stimulating the temporal language area in cases with motor-dominant or conduction aphasia. Therefore, this study focused on establishing passive language mapping for aphasic patients by combining real-time HGA mapping and CCEP recording during awake craniotomy. Hence, we hypothesized that it would be possible to identify and monitor brain function for the whole language network by passive listening and simple cortical stimulation. We thought that the combination should enable quick and less-invasive monitoring of language networks during awake craniotomy without the need for active patient cooperation.

\section{Methods \\ Patient Population}

Five patients, each with an intraaxial tumor affecting the frontotemporal language areas of the dominant hemisphere, underwent lesion resection by awake craniotomy at the Asahikawa Medical University between July 2014 and December 2014. The demographic data of these patients are listed in Table 1. The study was approved by our institutional review board, and written informed consent was obtained from each patient or their family before participation.

\section{Protocols for MRI}

Four patients underwent preoperative fMRI to evaluate if they could perform language tasks, but this was not needed in the patient in Case 1, who had severe conduction aphasia. All MRI studies were performed preoperatively with a 3-T whole-body MRI scanner with echo planar capabilities and a 32-channel surface coil (Discovery $750 \mathrm{~W}$; General Electric). We performed fMRI with a T2weighted echo planar imaging sequence. Each fMRI session consisted of 3 activation periods and 4 baseline (rest) periods lasting 2 minutes 12 seconds in total. The tasks used for patients were word reading (WR) and picture naming (PN). We used g.USBamp with a visual stimulation program (Guger Technologies OG) to present visual stimuli to the patients that were synchronized with scanning via hardware triggers.

Preprocessed data for each patient were analyzed with the standard general linear model approach by using boxcar predictors convolved with the canonical hemodynamic response function. Pixels with a z-score greater than 2.2 were considered to indicate fMRI activation (Dr. View, Infocom). For each patient, the fMRI results were coregistered and fused with the corresponding anatomical MR image of the patient's head, maximizing the mutual information between the data sets.

\section{Intraoperative ECoG Recording}

All patients underwent ECoG recording before, during, and after the lesion resection while receiving conscious sedation. We used two 20-channel ECoG grids with a 3 -mm electrode diameter and $10-\mathrm{mm}$ interelectrode distance (Unique Medical), together with 4-channel strip electrodes. The grids located the suspected frontal and temporal language areas by using the neuronavigation system according to the language-related fMRI results. One channel of the strip electrode, which reached the vertex of the frontal tip, was used as a reference, and the other was set as a ground. The grids and exposed brain surface were captured by a digital camera before resection. The ECoG data were recorded digitally by $1200-\mathrm{Hz}$ sampling with a 24-bit resolution and high oversampling to increase the signal-to-noise ratio, using a 256-channel g.HIamp biosignal amplifier (Guger Technologies OG). Electrode orientations were manually superimposed on the captured image. 
TABLE 1. Clinical and demographic data in 5 patients with intraaxial tumors

\begin{tabular}{cclll}
\hline Case No. & Age (yrs), Sex & Tumor Location & \multicolumn{1}{c}{ Symptoms } & Pathological Diagnosis \\
\hline 1 & $75, \mathrm{~F}$ & Lt frontal lobe & Motor aphasia & Glioblastoma \\
\hline 2 & $58, \mathrm{M}$ & Lt temporal lobe & Mild rt hemiparesis & Glioblastoma \\
\hline 3 & $59, \mathrm{~F}$ & Lt frontal lobe & Mild rt hemiparesis & Anaplastic oligoastrocytoma \\
\hline 4 & $28, \mathrm{M}$ & Bilat frontal lobe & Convulsive seizure & Diffuse astrocytoma \\
\hline 5 & $48, \mathrm{M}$ & Lt frontal lobe & No symptoms & Ganglioglioma \\
\hline
\end{tabular}

\section{Monitoring and Visualization of the Language Network Passive Listening Task for Real-Time HGA Mapping}

A native Japanese speaker read 4 different Japanese stories of at least 20 words in 15 seconds, which were recorded in the waveform audio file (WAVE) format. To activate the posterior language area, one of these stories was acoustically delivered to the patients while they were conscious. Linguistic stimuli were delivered at $100 \mathrm{~dB}$ via bilateral earphones, using a data-acquisition computer from g.HIamp, and using 1-KHz pure tones for $200 \mathrm{msec}$ as reference sounds. Each patient was first asked to relax without sound for 15 seconds (rest phase) and then passively listen to the delivered sounds (active phase). The rest and active phases were repeated alternately 3 times; thus, the measurement lasted for 90 seconds.

The ECoG data were analyzed by MATLAB R2012a (Mathworks) on the acquisition computer. After channels containing singular noise and epileptic discharges were excluded, we performed a common average reference to suppress common mode signals and calculated the baseline ECoG activity of each channel in the HGA between 80 and $160 \mathrm{~Hz}$. The patient was then instructed to perform each passive listening task, and the data processing was started to visualize the real-time ECoG activity. To obtain power averages across HGA, we standardized the power spectral density values for 3 of the 15-second ECoG data epochs that included baseline and active phases. A t-test was used to determine whether HGA values in the active phases were significantly different from those at baseline; for linguistic HGA mapping, we considered p values < 0.05 to be statistically significant after Bonferroni correction. The result of each task demonstrated red circles overlaying the electrodes, depending on the real-time statistical significance in the MATLAB environment. The significance value was represented by the circle diameter. All computations were performed in real time and updated at $20 \mathrm{~Hz}$.

\section{Protocol for Language Mapping With CCEP}

Based on linguistic HGA mapping, we stimulated the identified receptive language area on the temporal lesion. The electrical stimulus that we used comprised a constant, current square- wave pulse of $0.3 \mathrm{msec}$ at a frequency of $1 \mathrm{~Hz}$. Two adjacent electrodes were used for bipolar stimulation to achieve localized currents on the language cortices. Stimulation polarity was alternated to reduce the stimulus artifact. ${ }^{22}$ The current was given at $80 \%$ of the intensity that produced clinical signs or after-discharges during ECS; the maximum intensity was $15 \mathrm{~mA}$. The band-pass filter for data acquisition was between 1 and $1000 \mathrm{~Hz}$, with a sampling rate of $2000 \mathrm{~Hz}$ per channel. Responses were averaged using the stimulus onset as the trigger, with pre- and poststimulus periods of $100 \mathrm{msec}$ and $800 \mathrm{msec}$, respectively. In each session, we stimulated at least 5 points on the temporal region with or without HGA, and at least 2 trials of 30 responses were recorded to check the CCEP reproducibility. During recordings, participants were asked to keep their eyes closed and to remain relaxed. The electrical stimulus was delivered via the electrodes in an attempt to investigate the cortico-cortical connections from receptive language areas to frontal expressive language areas. This procedure did not need any specific tasks, and it detected CCEPs on the frontal electrode. Each CCEP epoch consisted of $50 \mathrm{msec}$ prestimulus and $150 \mathrm{msec}$ poststimulus time. Whereas CCEP epochs were averaged using the stimulation trigger, baseline epochs were averaged using a random software trigger that was not overlapping with the CCEP epochs. A Welch test, which is a derived t-test for populations with different variances, was used to check significant differences between the averaged baseline and CCEP epochs. The significance threshold was set to $p<0.05$ for a 2-tailed t-distribution.

\section{Electrocortical Stimulation Mapping}

Biphasic electrical pulses (frequency $50 \mathrm{~Hz}$, pulse duration $0.3 \mathrm{msec}$ ) were applied to the cortical sites of conscious patients using the same electrodes as for ECoG recording. The stimulus condition was bipolar, with $2 \mathrm{ECoG}$ channel pairs, and determined the stimulus intensity for each patient by first applying the stimulation at $3 \mathrm{~mA}$ and progressively increasing the current strength until the stimulation evoked neurological deficits or discharges. The stimulus current was increased to no more than $15 \mathrm{~mA}$ to avoid seizures. For language mapping during fMRI and ECoG recordings, we systematically used simple language tasks, including spontaneous speech, WR, and PN. The stimulus channel pairs were registered by the neuronavigation system.

For calculation of sensitivity and specificity we analyzed all 20 ECoG channels on the frontal and the temporal lobes, respectively. Although ECS was a bipolar setting, HGA and CCEPs were separately observed on each electrode.

All electrodes were classified into 4 groups according to whether each electrode involved HGA [HGA $(+)$ or HGA (-)] or CCEP [CCEP (+) or CCEP (-)] and/or whether each electrode was positive (+) or negative (-) on ECS. Using these 4 electrode groups, we obtained the sensitivity 
and specificity as follows: sensitivity $=$ HGA $(+) /$ ECS $(+)$ or CCEP $(+) /$ ECS $(+)$ and specificity $=$ HGA $(-) /$ ECS $(-)$ or CCEP $(-) / \operatorname{ECS}(-)$.

\section{Results}

\section{Patient Symptoms and Performance}

The patients' demographic data are presented in Table 1. Patients had no or little impairment of language-related function, except for the patient in Case 1, who had severe conduction aphasia. However, despite her neurological symptoms, this patient understood the presented words and pictures. None of the other 4 patients had any difficulties with the semantic tasks and underwent all mapping procedures, including HGA and CCEP recording and ECS mapping.

We started linguistic HGA mapping with the patients intubated, with a laryngeal mask airway. During awake craniotomy, the bispectral index (BIS) was maintained between 80 and 85 to obtain stable responses on the superior temporal region with no motion artifact. The mapping procedures consisted of 3 steps in the following order: linguistic HGA mapping, CCEP recording, and ECS mapping.

\section{Linguistic HGA Mapping}

All 5 patients passively listened to the sound stimuli. Pure tone evoked no significant HGA increase in any of the cases. In contrast, linguistic sounds resulted in multiple red bubbles appearing in the superior and middle temporal gyri. The spatial-temporal dynamics of the linguistic HGA bubbles were stable within 30 seconds of the sound delivery, and fewer than 8 bubbles (mean 4.45) remained for the posterior-superior temporal regions in all cases. The mean duration of the most active HGA was 2-9 seconds, and most bubbles were diminishing before the end of the third repetition. Linguistic HGA mapping indicated the receptive temporal language area in all 5 cases.

\section{The CCEP Technique for Language Mapping}

Single biphasic electrical currents were delivered via different electrode pairs to the positive or negative linguistic HGA areas. The 20-channel electrode covering the frontal language area recorded raw ECoG data with electrical triggers.

In the 5 patients undergoing awake craniotomy with the same BIS level, CCEPs consisted of one peak in 4 cases and two peaks in 1 case. The mean latency of the main peak was $55.4 \pm 21.4 \mathrm{msec}$, and the mean amplitude of the main peak was $58.02 \pm 30.6 \mu \mathrm{V}$. The main peaks of CCEPs mostly appeared on the posterior part of the inferior and middle frontal gyri, whereas no CCEPs appeared by stimulating any of the bubble-negative temporal areas, in all cases.

\section{Comparison Among HGA, CCEP, and ECS Mappings}

The BIS was increased beyond 90 to keep patients alert and to allow them to perform semantic tasks for ECS mapping only. Although we achieved minimal ECS mapping in Case 1 due to her severe aphasia, we resected the lesion while preserving the frontal CCEPs. In the other 4 cases, detailed ECS mapping showed spatial distribution of language-related functions.

For the temporal language areas on linguistic HGA mapping, ECS consistently induced speech arrest or total aphasia. The sensitivity and specificity of linguistic HGAECS mapping for the temporal language area were $93.8 \%$ and $95 \%$, respectively, compared with $93.8 \%$ and $83 \%$, respectively, for CCEP-ECS mapping in the frontal area. After the lesion resection, CCEPs were preserved in all cases.

\section{Postoperative Course}

After tumor resection, aphasia had not worsened in Case 1, but the patients in Cases 2 and 3 each suffered from transient naming difficulty for 2 weeks. The postoperative courses of other 2 patients (Cases 4 and 5) were uneventful.

\section{Representative Cases \\ Case 2}

This 58-year-old man had mild right-sided hemiparesis with no language deficit for 2 months. The MRI sequence obtained with Gd-DTPA enhancement revealed a lesion in the left temporal tip (Fig. 1A), and T2-weighted images showed a small hyperintense spot in the left centrum semiovale. The surgical strategy was to resect the entire area of enhancement, preserving temporal language functions. After craniotomy, the patient was able to communicate with staff and understood verbal commands. Linguistic HGA mapping demonstrated 3 channels with significant HGA increase in the superior temporal gyrus (Fig. 1B). The electrical stimuli to an HGA-positive electrode pair consistently induced CCEPs peaking at $23.3 \mathrm{msec}$ on 4 electrodes (F4, F5, F9, and F10) on the frontal lobe (Figs. $1 \mathrm{C}$ and 2). The ECS to the linguistic HGA-positive and frontal CCEP-positive electrodes induced total aphasia and paranomia, respectively. For example, stimulation to T15-20 and T14-20 induced severe aphasia. The ECS to the frontal electrodes (F3-9, F4-9, F5-9, F9-13, and F9-14) evoked complex symptoms such as paranomia, anomia, or total aphasia (Fig. 1D). We confirmed that no neurological deficit was evoked by ECS with other channel pairs. The sensitivity and specificity of HGA mapping were $100 \%$ compared with ECS mapping. The CCEP mapping produced sensitivity and specificity values of $75 \%$ and $81 \%$, respectively. The patient suffered from transient aphasia for 8 days after surgery.

\section{Case 3}

This 59-year-old woman had mild right-sided hemiparesis with no language deficit. An MRI study demonstrated small enhancement spots in a diffuse lesion with hyperintensity in the left frontal lobe on T2-weighted images (Fig. 3A). The surgical strategy was to perform maximal tumor resection while preserving the frontal language function. In the initial 30 minutes of awake craniotomy, the patient was drowsy and found it difficult to follow simple commands at a BIS of 80. Linguistic HGA mapping demonstrated 4 active channels on the superior temporal gyrus only, and showed characteristic HGA dynam- 

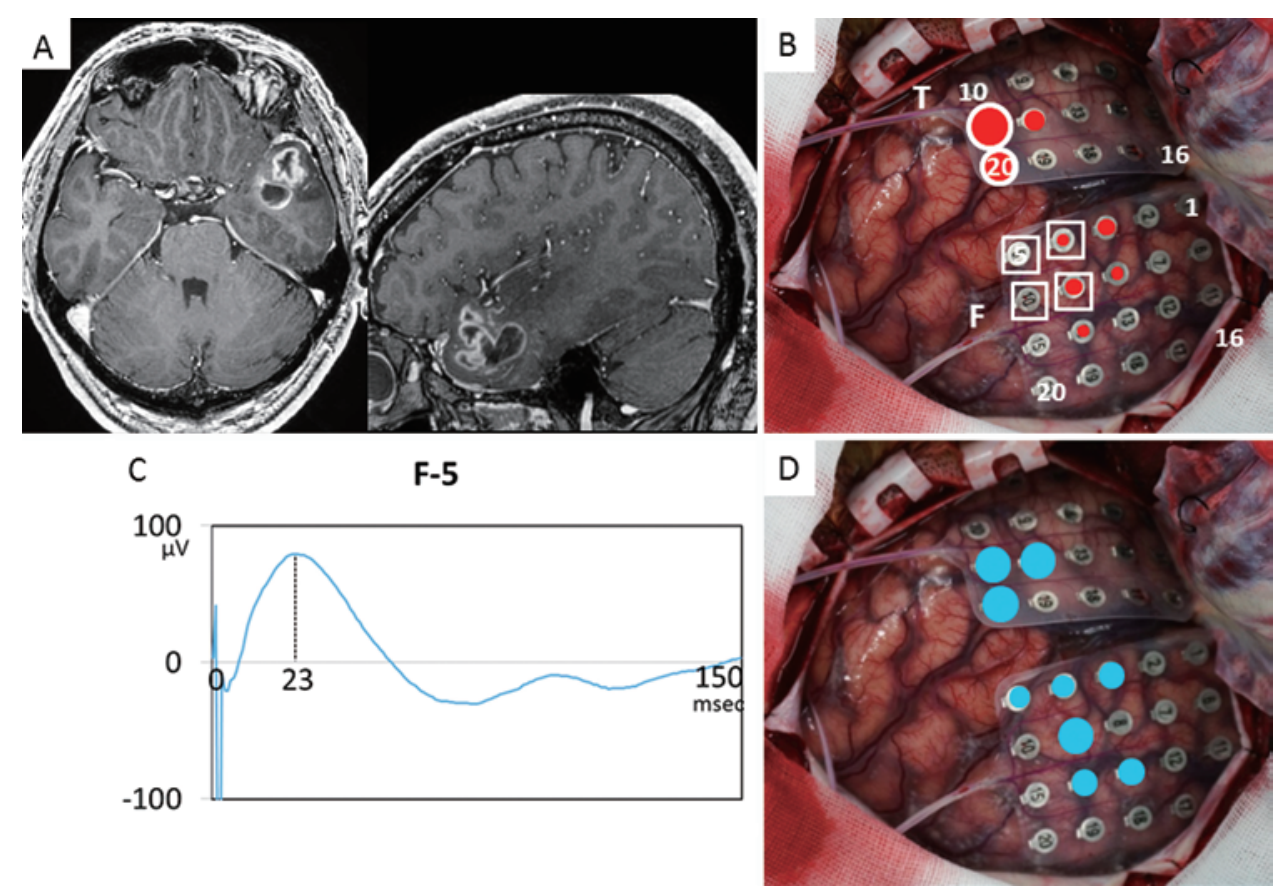

FIG. 1. Case 2. A patient with a glioblastoma of the left temporal lobe. A: A lesion with ringlike enhancement is demonstrated on the preoperative MRI sequences. B: Photograph of the exposed brain surface before resection. Red bubbles indicate active channels with significant changes of HGA with linguistic sounds. Linguistic-HGA appears dominantly in the superior temporal gyrus. White circles represent stimulus channel pairs for CCEPs, and white squares represent active channels with significant CCEPs. The CCEPs appear only on the frontal lobe. C: Representative CCEP waveform of F5 peaking at 23 msec after the stimulation. D: Photograph showing ECS mapping. Blue bubbles indicate channels that induced language-related deficits. $F=$ frontal lobe; $\mathrm{T}$ = temporal lobe.

ics (Fig. 3B). Linguistic HGA rapidly increased within 10 seconds of delivering the linguistic stimuli and decreased quickly. Whereas the stimulation to the T15 and T20 pair evoked larger CCEPs on F2 and F3, stimulation of T19 and T20 induced CCEPs on F2, F3, and F5 (Fig. 3C). The ECS to T15-20 and T19-20 induced total aphasia and severe paranomia, respectively. The ECS to F2-3, F3-4, and $\mathrm{F} 4-5$ provoked speech hesitation or naming difficulty (Fig. 3D). For HGA mapping, the sensitivity and specificity were $75 \%$ and $100 \%$, respectively, whereas those for CCEP recording were $100 \%$ and $89 \%$, respectively. Based on ECS mapping, we resected the frontal lesion, sparing the language cortices. After surgery, the patient suffered from transient naming difficulty for 2 weeks.

\section{Case 5}

This 48-year-old man had experienced a generalized seizure 1 month earlier, and although he had no neurological deficit, MRI uncovered a ringlike enhancement lesion in the left insular cortex (Fig. 4A). Under awake craniotomy, we achieved linguistic HGA, frontal CCEPs, and ECS mapping (Fig. 4B-D). Whereas the sensitivity and specificity of linguistic HGA were $100 \%$ and $92 \%$, respectively, frontal CCEPs showed a sensitivity of $100 \%$ and a specificity of $81 \%$.

The combination of linguistic HGA and frontal CCEPs needed no patient cooperation or effort. The overall sensitivity and specificity in 4 patients were $93.8 \%$ and $89 \%$, respectively (Table 2 ).

\section{Discussion}

This report described a passive mapping procedure that facilitates intraoperative language mapping with no requirement for patients to perform semantic tasks. The procedure consisted of 2 novel steps: 1) linguistic HGA mapping with linguistic sounds; and 2) CCEP with electrical pulses to the temporal receptive language area. Linguistic HGA mapping quickly indicated the language-related area on the temporal lobe, with high sensitivity and specificity. Electrical stimulation to the identified temporal receptive language area by linguistic HGA mapping evoked CCEPs in the frontal lobe. The ECS mapping of the areas with CCEPs evoked functional deficits of language-related functions with high sensitivity. This study demonstrates that the proposed novel technique was reliable for functional brain mapping of higher complex functions without the need for active tasks, which we termed "passive" mapping in awake craniotomy.

Over the last decade, growing attention has been given to the study of brain oscillatory activity, including eventrelated synchronization (ERS) and event-related desynchronization (ERD) in human ECoG. The ERS and ERD can be observed in various frequency ranges, and HGA or ERS in the broad-band, high-gamma-frequency range has been among the other most intensively studied methods. Previous electrophysiology reports ${ }^{3}$ suggest strong correlations between HGA and various functional domains, including auditory, $5,8,36$ and language., ${ }^{6,20,37}$ Gamma oscillation may be induced by synchronized activity of $\gamma$ - 


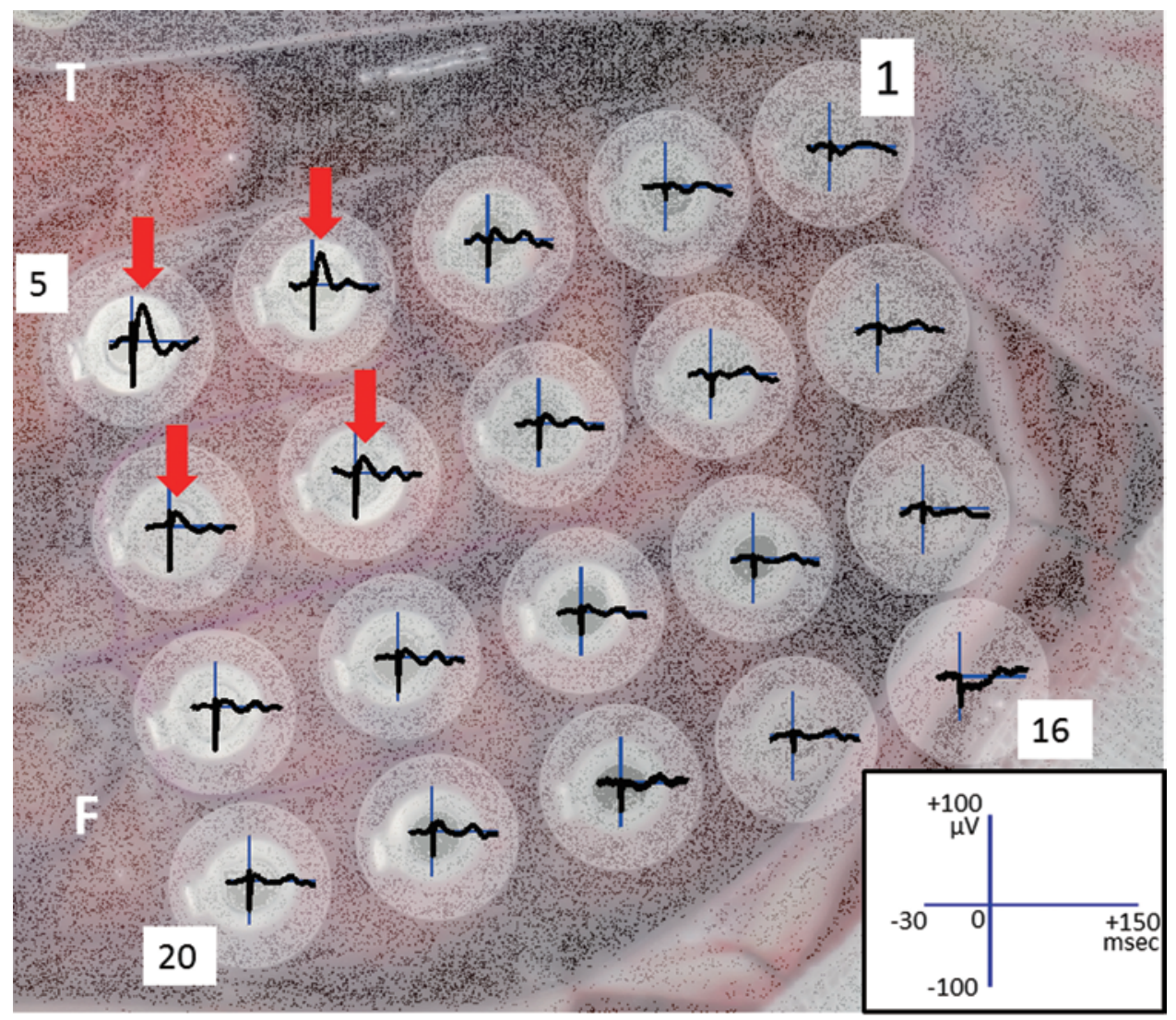

FIG. 2. Intraoperative recording of CCEPs in the left frontal lobe. Channels 4, 5, 9, and 10 show obvious CCEPs peaking at approximately $24 \mathrm{msec}$ (red arrows). There are peaks on the posterior part of the inferior frontal gyrus. Black lines indicate waveforms by electrical stimuli to the receptive temporal language area.

aminobutyric acidergic (GABAergic) interneurons in the population network, playing an important role in controlling the timing of action potential generation in pyramidal cells. ${ }^{4,12,13,35}$ Recently, several works have revealed that gamma-band power is positively correlated with neuronal firing rates, ${ }^{21,23,26}$ supporting the view that HGA may be a strong candidate for an index of local cortical activation. Taken together, the analysis of HGA dynamics appears to be one of the most powerful approaches for studying the mechanisms of language functions. Kunii et al. reported on the normalization of subdural electrodes in 21 epileptic patients for visualization of typical HGA dynamics. ${ }^{17}$ They integrated the HGAs of all patients onto 1 template brain and compared the results of HGA dynamics for 3 language tasks. They found that significantly increased HGA appeared within $500 \mathrm{msec}$ after the onset of the sound stimuli in the lateral aspect of the temporal lobe. In addition, the Japanese stories in our study that included key linguistic sounds significantly increased HGA in the posterior-lateral temporal language area compared with pure tones.

There have been 2 reports about real-time intraoperative HGA mapping, which stressed 2 key advantages. First, it is noninvasive with no seizure risk. Second, it requires less time for cortical mapping than existing techniques. However, these previous reports still relied on active tasks, such as hand movement, WR, and PN, and therefore were still dependent on patient cooperation. In the present study, we used 2 different sound stimuli to identify the receptive temporal language functions, and found that linguistic sounds consisting of various tones and pitches activated the lateral aspect of the superior and middle temporal gyri. Linguistic HGA mapping was completely passive, allowing rapid assessment of HGA dynamics. Moreover, the sensitivity and specificity of real-time HGA mapping for the receptive temporal language area were $93.8 \%$ and 95\%, respectively. Linguistic HGA should therefore be a good indicator of receptive-language function.

The CCEP technique provides a unique opportunity to track in vivo cortico-cortical connections electrophysiologically by stimulating part of the cortex through subdural electrodes and recording evoked cortical potentials derived from the remote cortical regions. This method has been reported to delineate the cortico-cortical networks involved in language systems, ${ }^{19,22,27}$ with 2 reports about intraoperative use of CCEPs for functional language monitoring. Saito et al. found that a patient's language symptoms worsened when CCEPs disappeared during operations and suggested that CCEP profiles may reflect postsurgical neurological symptoms. ${ }^{29}$ Matsumoto et al. simultaneously detected CCEPs from both the frontal and temporal language centers by stimulating deep resected areas by the CCEP technique's bidirectional responses. ${ }^{22}$ Based on these facts, it appears appropriate to record CCEPs in either cortex for 

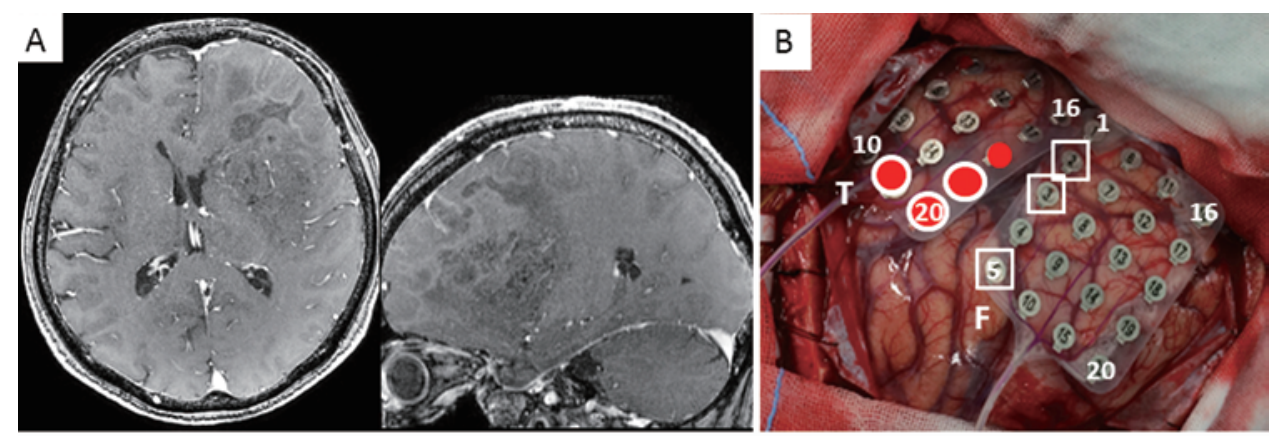

$\mathrm{C}$

F-5
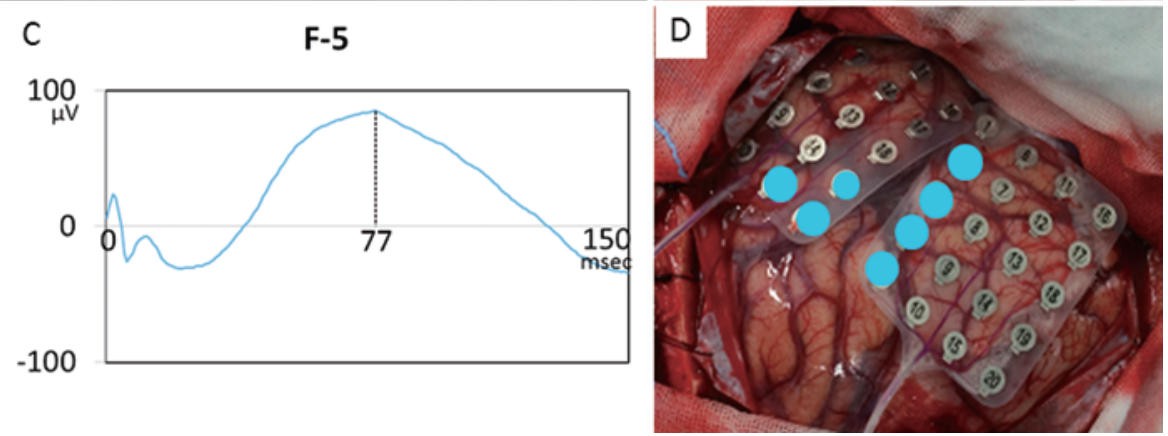

FIG. 3. Case 3. A patient with an anaplastic oligoastrocytoma of the left frontal lobe. A: Preoperative MRI showing a nonhomogeneous lesion, including cyst, and mild enhancement. B: Photograph of the exposed brain surface before resection. Red bubbles indicate active channels with significant changes of HGA with linguistic sounds. Linguistic-HGA appears dominantly in the superior temporal gyrus. White circles represent stimulus channel pairs for CCEPs, and white squares represent active channels with significant CCEPs. The CCEPs appear only on the inferior frontal lobe. C: Representative CCEP waveform of F5 peaking at 77 msec after the stimulation. D: Photograph showing ECS mapping. Blue bubbles indicate channels that induced language-related deficits.
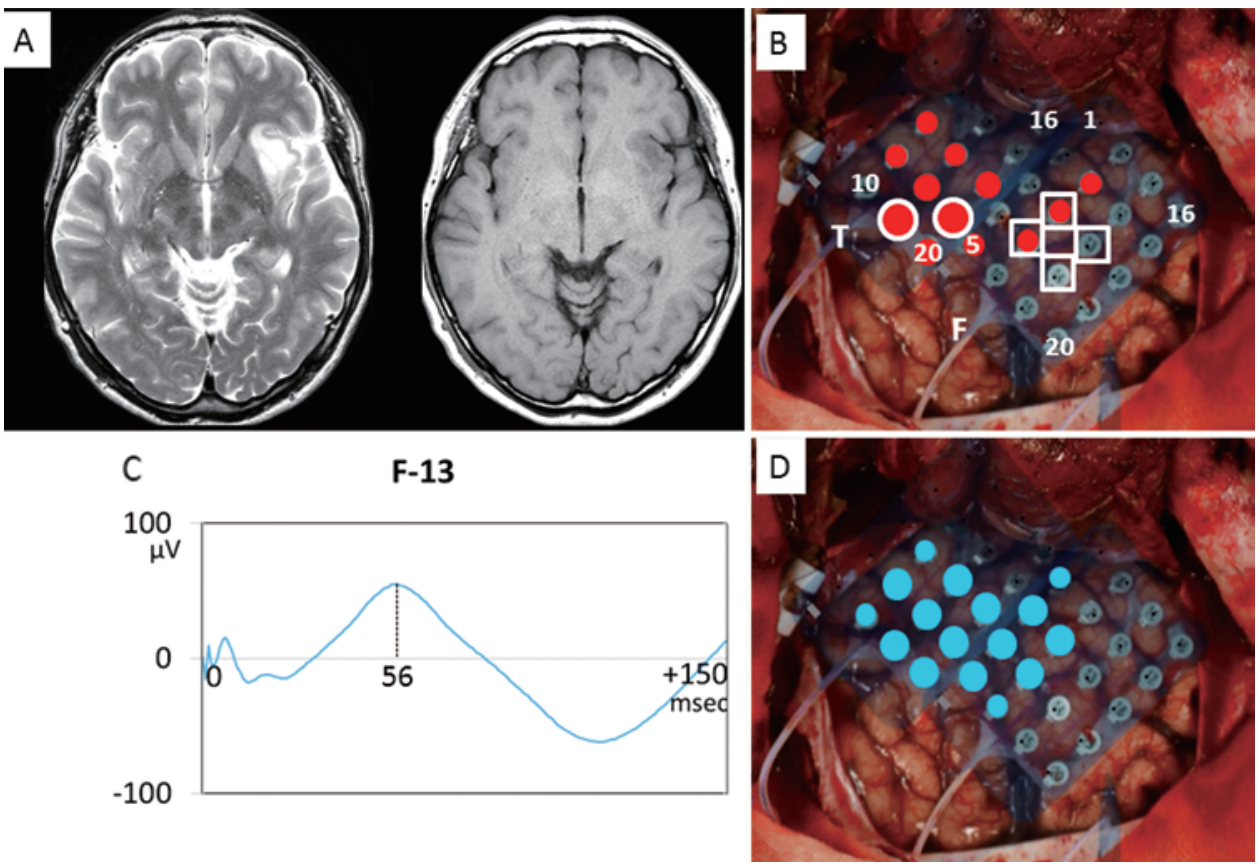

FIG. 4. Case 5. A patient with a ganglioglioma of the left insular cortex. A: Preoperative MRI showing a hyperintense lesion on T2weighted image without enhancement. B: Photograph of the exposed brain surface before resection. Red bubbles indicate active channels with significant changes of HGA with linguistic sounds. Linguistic-HGA appears dominantly in the superior and middle temporal gyri. White circles represent a stimulus channel pair for CCEPs, and white squares represent active channels with significant CCEPs. The CCEPs appear only on the frontal lobe. C: Representative CCEP waveform of F13 peaking at 56 msec after the stimulation. D: Photograph showing ECS mapping. Blue bubbles indicate channels that induced language-related deficits. 
TABLE 2. Sensitivity and specificity of CCEPs, HGA, and ECS for brain mapping in 5 patients with intraaxial tumors

\begin{tabular}{ccccc}
\hline \multirow{2}{*}{$\begin{array}{c}\text { Nase } \\
\text { No. }\end{array}$} & \multicolumn{2}{c}{ Temporal HGA-ECS } & \multicolumn{2}{c}{ Frontal CCEP-ECS } \\
\cline { 2 - 5 } & Sensitivity (\%) & Specificity (\%) & Sensitivity (\%) & Specificity (\%) \\
\hline 1 & NP & NP & NP & NP \\
\hline 2 & 100 & 100 & 75 & 81 \\
\hline 3 & 75 & 100 & 100 & 89 \\
\hline 4 & 100 & 88 & 100 & 81 \\
\hline 5 & 100 & 92 & 100 & 81 \\
\hline
\end{tabular}

$\mathrm{NP}=$ not performed.

functional mapping of the language network. However, the existing reports were still dependent on patient cooperation to find appropriate stimulus sites for CCEPs by ECS mapping or functional MRI. The novel technique proposed in this study does not require any patient corporation or effort, and we believe that such passive mapping will be applicable to any patient with language deficits or those who are unable to cooperate adequately.

In this report, 4 patients showed only one peak at approximately $55.3 \mathrm{msec}$ during awake craniotomy. According to the original report on CCEPs in epileptic patients, the waveforms typically consisted of two peaks at approximately 30 and $110 \mathrm{msec}$; however, intraoperative CCEP recording by Saito et al. ${ }^{29}$ did observe just one main peak at approximately $50 \mathrm{msec}$. Given that the original investigation was performed at the bedside, with no effect from anesthesia, it is plausible that the sensitivity and excitability of the CNS may have been different from that during awake craniotomy.

The precise generator mechanisms of CCEPs remain unknown. The general assumption is that on cortical stimulation, orthodromic excitation of cortico-cortical projection neurons occurs through direct depolarization of the initial segment, with concurrent synaptic excitation in the local circuit. With oligosynaptic excitation of the corticocortical projection neurons, the impulse travels through cortico-cortical projections to the target cortex with some jitter, and then generates a relatively blunt potential peak. There was additional potential in the indirect corticosubcortico-cortical pathways, which showed peaks with various latencies. ${ }^{9}$ Nevertheless, various hypotheses exist about the origins of CCEPs, so assigning peak CCEPs remains uncertain.

Sanai et al. proposed a new mapping procedure, which they called "negative" mapping. According to their surgical experiences with 145 patients, they concluded that aggressive lesion resection could be safely achieved without producing language deficits. ${ }^{33}$ This was because they found no positive language sites, even with limited cortical exposure. Consequently, negative mapping is now widely accepted, although appropriate ECS remains indispensable for making critical resection decisions. Because our primary aim was to perform functional mapping quickly in patients with language deficits, we focused on detecting neurophysiological signals directly from the brain surface without the requirement for active tasks. Thus, we believe that passive mapping, consisting of linguistic HGA and frontal CCEPs, is a valuable alternative to negative mapping. We expect that this novel technique will become established as one of the mapping options for identifying functional areas in patients with neurological deficits. Passive mapping revealed characteristic electrophysiological dynamics in the frontal and temporal language areas, but further verification with a larger sample size is needed to verify our data, clarify the variations in spatial and temporal dynamics of HGA and CCEPs, and investigate clinically significant interpatient differences. We believe that the proposed passive techniques have great potential for intraoperative neurophysiological monitoring in neurosurgery.

\section{Conclusions}

We demonstrated the clinical impact of passive mapping that detects electrophysiological signals directly from the brain surface. While real-time HGA mapping with linguistic stimuli rapidly indicated the receptive temporal language area, CCEPs appeared on the frontal language cortices. The ECS mapping confirmed the reliability of passive mapping with high sensitivity and specificity. This technique can make functional brain mapping more reliable and enable us to formulate rational and objective operation strategies. Furthermore, passive mapping sheds light on the underlying physiological mechanisms related to language in the human brain. However, a prospective study with a larger sample is needed to verify our results.

\section{Acknowledgments}

This work was supported in part by a Grant-in-Aid for Scientific Research (B) No. 24390337 (2012-2015) and a Grant-in-Aid for Exploratory Research No. 26670633 (2014-2016) from the Ministry of Education, Culture, Sports, Science and Technology (MEXT) and JSPS KAKENHI Grant Number 15H01657, as well as the European Union FP7 Integrated Project VERE No. 257695 and the EU project "High Profile" No. 269356.

\section{References}

1. Basser PJ, Pajevic S: A normal distribution for tensor-valued random variables: applications to diffusion tensor MRI. IEEE Trans Med Imaging 22:785-794, 2003

2. Bizzi A, Blasi V, Falini A, Ferroli P, Cadioli M, Danesi U, et al: Presurgical functional MR imaging of language and motor functions: validation with intraoperative electrocortical mapping. Radiology 248:579-589, 2008

3. Cardin JA, Carlén M, Meletis K, Knoblich U, Zhang F, Deisseroth K, et al: Driving fast-spiking cells induces gamma rhythm and controls sensory responses. Nature 459:663667,2009

4. Cobb SR, Buhl EH, Halasy K, Paulsen O, Somogyi P: Synchronization of neuronal activity in hippocampus by individual GABAergic interneurons. Nature 378:75-78, 1995

5. Crone NE, Boatman D, Gordon B, Hao L: Induced electrocorticographic gamma activity during auditory perception. Brazier Award-winning article, 2001. Clin Neurophysiol 112:565-582, 2001

6. Crone NE, Hao L, Hart J Jr, Boatman D, Lesser RP, Irizarry $\mathrm{R}$, et al: Electrocorticographic gamma activity during word production in spoken and sign language. Neurology 57:2045-2053, 2001

7. Duffau H, Moritz-Gasser S, Gatignol P: Functional outcome after language mapping for insular World Health Organiza- 
tion Grade II gliomas in the dominant hemisphere: experience with 24 patients. Neurosurg Focus 27(2):E7, 2009

8. Edwards E, Soltani M, Deouell LY, Berger MS, Knight RT: High gamma activity in response to deviant auditory stimuli recorded directly from human cortex. J Neurophysiol 94:4269-4280, 2005

9. Entz L, Tóth E, Keller CJ, Bickel S, Groppe DM, Fabó D, et al: Evoked effective connectivity of the human neocortex. Hum Brain Mapp 35:5736-5753, 2014

10. Fernández G, Specht K, Weis S, Tendolkar I, Reuber M, Fell $\mathrm{J}$, et al: Intrasubject reproducibility of presurgical language lateralization and mapping using fMRI. Neurology 60:969975, 2003

11. FitzGerald DB, Cosgrove GR, Ronner S, Jiang H, Buchbinder $\mathrm{BR}$, Belliveau JW, et al: Location of language in the cortex: a comparison between functional MR imaging and electrocortical stimulation. AJNR Am J Neuroradiol 18:1529-1539, 1997

12. Fries P, Nikolić D, Singer W: The gamma cycle. Trends Neurosci 30:309-316, 2007

13. Hasenstaub A, Shu Y, Haider B, Kraushaar U, Duque A, McCormick DA: Inhibitory postsynaptic potentials carry synchronized frequency information in active cortical networks. Neuron 47:423-435, 2005

14. Kamada K, Todo T, Masutani Y, Aoki S, Ino K, Morita A, et al: Visualization of the frontotemporal language fibers by tractography combined with functional magnetic resonance imaging and magnetoencephalography. J Neurosurg 106:90-98, 2007

15. Kamada K, Todo T, Masutani Y, Aoki S, Ino K, Takano T, et al: Combined use of tractography-integrated functional neuronavigation and direct fiber stimulation. J Neurosurg 102:664-672, 2005

16. Kamada K, Todo T, Ota T, Ino K, Masutani Y, Aoki S, et al: The motor-evoked potential threshold evaluated by tractography and electrical stimulation. J Neurosurg 111:785-795, 2009

17. Kunii N, Kamada K, Ota T, Greenblatt RE, Kawai K, Saito $\mathrm{N}$ : The dynamics of language-related high-gamma activity assessed on a spatially-normalized brain. Clin Neurophysiol 124:91-100, 2013

18. Kunii N, Kamada K, Ota T, Kawai K, Saito N: A detailed analysis of functional magnetic resonance imaging in the frontal language area: a comparative study with extraoperative electrocortical stimulation. Neurosurgery 69:590-597, 2011

19. Lacruz ME, García Seoane JJ, Valentin A, Selway R, Alarcón G: Frontal and temporal functional connections of the living human brain. Eur J Neurosci 26:1357-1370, 2007

20. Mainy N, Kahane P, Minotti L, Hoffmann D, Bertrand O, Lachaux JP: Neural correlates of consolidation in working memory. Hum Brain Mapp 28:183-193, 2007

21. Manning JR, Jacobs J, Fried I, Kahana MJ: Broadband shifts in local field potential power spectra are correlated with singleneuron spiking in humans. J Neurosci 29:13613-13620, 2009

22. Matsumoto R, Nair DR, LaPresto E, Najm I, Bingaman W, Shibasaki H, et al: Functional connectivity in the human language system: a cortico-cortical evoked potential study. Brain 127:2316-2330, 2004

23. Nir Y, Fisch L, Mukamel R, Gelbard-Sagiv H, Arieli A, Fried I, et al: Coupling between neuronal firing rate, gamma LFP, and BOLD fMRI is related to interneuronal correlations. Curr Biol 17:1275-1285, 2007

24. Ogawa H, Kamada K, Kapeller C, Hiroshima S, Prueckl R, Guger C: Rapid and minimum invasive functional brain mapping by real-time visualization of high gamma activity during awake craniotomy. World Neurosurg 82:912.e1-912.e10, 2014

25. Pouratian N, Bookheimer SY, Rex DE, Martin NA, Toga AW: Utility of preoperative functional magnetic resonance imag- ing for identifying language cortices in patients with vascular malformations. J Neurosurg 97:21-32, 2002

26. Ray S, Crone NE, Niebur E, Franaszczuk PJ, Hsiao SS: Neural correlates of high-gamma oscillations $(60-200 \mathrm{~Hz})$ in macaque local field potentials and their potential implications in electrocorticography. J Neurosci 28:11526-11536, 2008

27. Rosenberg DS, Mauguière F, Catenoix H, Faillenot I, Magnin M: Reciprocal thalamocortical connectivity of the medial pulvinar: a depth stimulation and evoked potential study in human brain. Cereb Cortex 19:1462-1473, 2009

28. Rutten GJ, Ramsey NF, van Rijen PC, Noordmans HJ, van Veelen CW: Development of a functional magnetic resonance imaging protocol for intraoperative localization of critical temporoparietal language areas. Ann Neurol 51:350-360, 2002

29. Saito T, Tamura M, Muragaki Y, Maruyama T, Kubota Y, Fukuchi S, et al: Intraoperative cortico-cortical evoked potentials for the evaluation of language function during brain tumor resection: initial experience with 13 cases. J Neurosurg 121:827-838, 2014

30. Sanai N, Berger MS: Intraoperative stimulation techniques for functional pathway preservation and glioma resection. Neurosurg Focus 28(2):E1, 2010

31. Sanai N, Berger MS: Operative techniques for gliomas and the value of extent of resection. Neurotherapeutics 6:478486, 2009

32. Sanai N, Berger MS: Recent surgical management of gliomas. Adv Exp Med Biol 746:12-25, 2012

33. Sanai N, Mirzadeh Z, Berger MS: Functional outcome after language mapping for glioma resection. N Engl J Med 358: $18-27,2008$

34. Scarabino T, Giannatempo GM, Popolizio T, Tosetti M, d'Alesio V, Esposito F, et al: 3.0-T functional brain imaging: a 5-year experience. Radiol Med (Torino) 112:97-112, 2007

35. Traub RD, Cunningham MO, Gloveli T, LeBeau FE, Bibbig A, Buhl EH, et al: GABA-enhanced collective behavior in neuronal axons underlies persistent gamma-frequency oscillations. Proc Natl Acad Sci U S A 100:11047-11052, 2003

36. Trautner P, Rosburg T, Dietl T, Fell J, Korzyukov OA, Kurthen $\mathrm{M}$, et al: Sensory gating of auditory evoked and induced gamma band activity in intracranial recordings. Neuroimage 32:790-798, 2006

37. Wu HC, Nagasawa T, Brown EC, Juhasz C, Rothermel R, Hoechstetter K, et al: $\gamma$-oscillations modulated by picture naming and word reading: intracranial recording in epileptic patients. Clin Neurophysiol 122:1929-1942, 2011

38. Yamao Y, Matsumoto R, Kunieda T, Arakawa Y, Kobayashi $\mathrm{K}$, Usami K, et al: Intraoperative dorsal language network mapping by using single-pulse electrical stimulation. Hum Brain Mapp 35:4345-4361, 2014

\section{Disclosures}

The authors report no conflict of interest concerning the materials or methods used in this study or the findings specified in this paper.

\section{Author Contributions}

Conception and design: Kamada, Ritaccio. Acquisition of data: Kamada, Tamura, Ogawa, Kapeller, Prueckl, Anei. Analysis and interpretation of data: Tamura, Ogawa, Kapeller, Prueckl, Takeuchi. Drafting the article: Kamada. Administrative/technical/ material support: Kamada, Guger. Study supervision: Kamada.

\section{Correspondence}

Kyousuke Kamada, Department of Neurosurgery, Asahikawa Medical University, 2-1, Midorigaoka-Higashi, Asahikawa, Hokkaido 078-8510, Japan. email: kamady-k@umin.ac.jp. 